\title{
PLANAR IMAGES OF DECOMPOSABLE CONTINUA
}

\section{Charles L. Hagopian}

\begin{abstract}
A nondegenerate metric space that is both compact and connected is called a continuum. In this paper it is proved that if $M$ is a continuum with the property that for each indecomposable subcontinuum $H$ of $M$ there is a continuum $K$ in $M$ containing $H$ such that $K$ is connected im kleinen at some point of $H$ and if $f$ is a continuous function on $M$ into the plane, then the boundary of each complementary domain of $f(M)$ is hereditarily decomposable. Consequently, if $M$ is a continuum in Euclidean $n$-space that does not contain an indecomposable continuum in its boundary, then no planar continuous image of $M$ has an indecomposable continuum in the boundary of one of its complementary domains.
\end{abstract}

For a given set $Z$, the closure and the boundary of $Z$ are denoted by $\mathrm{Cl} Z$ and Bd $Z$ respectively. The union of the elements of $Z$ is denoted by St $Z$.

THEOREM 1. If $X$ is a continuum in a 2-sphere $S$ and $I$ is an indecomposable subcontinuum of $X$ that is contained in the boundary of a complementary domain of $X$, then every subcontinuum of $X$ that contains a nonempty open subset of I contains $I$.

Proof. Let $D$ be a complementary domain of $X$ such that $I \subset \mathrm{Bd} D$, and let $X^{\prime}=S-D$. By Theorem 1 of [1], every subcontinuum of $X^{\prime}$, and hence every subcontinuum of $X$, which contains a nonempty open subset of $I$ contains $I$.

Definition. An indecomposable subcontinuum $I$ of a continuum $X$ is said to be terminal in $X$ if there exists a composant $C$ of $I$ such that each subcontinuum of $X$ that meets both $C$ and $X-I$ contains $I$.

Theorem 2. Suppose $X$ is a plane continuum, $I$ is an indecomposable subcontinuum of $X$, and each subcontinuum of $X$ that contains a nonempty open subset of $I$ contains $I$. Then $I$ is terminal in $X$.

Proof. Suppose there exists a collection $E$ of continua in $X$ such that for each composant $C$ of $I$ there is an element of $E$ that meets both $C$ and $X-I$ and does not contain $I$. Let $\left\{U_{n}\right\}$ be the elements of a countable base (for the topology on the plane) that intersect $I$. For each positive integer $n$, let $P_{n}$ be the set consisting of all components $Q$ of $I-U_{n}$ such that $Q$ meets an element of $E$ that is con- 
tained in $X-\mathrm{Cl} U_{n}$. Since $I=\mathrm{U}_{n=1}^{\infty} \mathrm{St} P_{n}$, for some integer $n$, the set St $P_{n}$ is a second category subset of $I$. Let $L$ be the set consisting of all elements $B$ of $P_{n}$ such that there exists a subcontinuum $F$ of an element of $E$ contained in $X-\mathrm{Cl} U_{n}$ with the property that $F$ meets both $B$ and $X-I$ and does not intersect $I-B$. According to a theorem of Kuratowski's [3], St $L$ is a first category subset of $I$. Let $J$ denote the set of all elements $H$ of $E$ such that $H$ is contained in $X-\mathrm{Cl} U_{n}$ and meets an element of $P_{n}-L$. Define $R$ to be the union of all components of St $\left(J \cup P_{n}\right)$ that intersect the set St $J$. Each element of $J$ meets three elements of $P_{n}$. Hence each component of $R$ contains a triod. It follows that the components of $R$ are countable. Since $\operatorname{St}\left(P_{n}-L\right)$ is a second category subset of $I$ that is contained in $R$, there exists a component $T$ of $R$ such that Cl $T$ contains a nonempty open subset of $I$. But since $\mathrm{Cl} T$ is a continuum in $X-U_{n}$, this is a contradiction. Hence $I$ is terminal in $X$.

THEOREM 3. Suppose $M$ is a continuum with the property that for each indecomposable subcontinuum $H$ of $M$ there is a continuum $K$ in $M$ containing $H$ such that $K$ is connected im kleinen at some point of $H$ and $f$ is a continuous function on $M$ into the plane. Then the boundary of each complementary domain of $f(M)$ is hereditarily decomposable.

Proof. Suppose a complementary domain of $f(M)$ contains an indecomposable continuum $I$ in its boundary. According to Theorems 1 and $2, I$ is terminal in $f(M)$. Hence there exists a composant $C$ of $I$ such that each subcontinuum of $f(M)$ that meets both $C$ and $f(M)-I$ contains $I$. Let $p$ be a point of $f^{-1}(C)$. Define $Z$ to be the $p$-component of $f^{-1}(I)$. As in the proof of Theorem 2 of [2], $f(Z)=I$.

Let $A$ be a composant of $I$ distinct from $C$. There exists a continuum $H$ in $Z$ such that $f(H)$ meets $A$ and $C$, and no proper subcontinuum of $H$ has an image under $f$ that meets both $A$ and $C$. Note that $f(H)=I$ and $H$ is indecomposable. There is a continuum $K$ in $M$ containing $H$ that is connected im kleinen at some point of $H$. Hence there exists a continuum $W$ in $K$ whose interior (relative to $K$ ) meets $H$ such that $f(W)$ does not contain $I$. Each composant of $H$ meets $W$.

Let $x$ be a point of $H \cap f^{-1}(C)$. Since the $x$-composant of $H$ intersects $W$, it follows that $f(W)$ is contained in $C$. Let $y$ be a point of $H \cap f^{-1}(A)$. There exists a proper subcontinuum $Y$ of $H$ that contains $y$ and meets $W$. Since $f(Y)$ meets both $A$ and $C$, this is a contradiction. Hence the boundary of each complementary domain of $f(M)$ is hereditarily decomposable. 
COROLlaRY 1. If a continuous image of a hereditarily decomposable continuum lies in the plane, then the boundary of each of its complementary domains is hereditarily decomposable.

CoRollary 2. If $M$ is a continuum in Euclidean $n$-space that does not contain an indecomposable continuum in its boundary and $f$ is a continuous function on $M$ into the plane, then the boundary of each complementary domain of $f(M)$ is hereditarily decomposable.

The author gratefully acknowledges conversations about these results with Professors E. E. Grace and F. B. Jones.

\section{REFERENCES}

1. C. L. Hagopian, A fixed point theorem for plane continua, Bulletin Amer. Math. Soc., 77 (1971), 351-354.

2. $-\lambda$ connected plane continua, to appear.

3. K. Kuratowski, Sur une condition qui caractérise les continus indecomposables, Fundamenta Math., 14 (1929), 116-117.

Received June 17, 1971 and in revised form September, 7, 1971.

Sacramento State College

AND

ARizona State University 
